\title{
Determining the role of IL-4 induced neuroinflammation in microglial activity and amyloid- $\beta$ using BV2 microglial cells and APP/PS1 transgenic mice
}

\author{
Clare H Latta ${ }^{1,5}$, Tiffany L Sudduth ${ }^{1}$, Erica M Weekmann ${ }^{1,2}$, Holly M Brothers ${ }^{1}$, Erin L Abner ${ }^{1,3}$, Gabriel J Popa ${ }^{4}$, \\ Michael D Mendenhall ${ }^{4}$, Floracita Gonzalez-Oregon ${ }^{1,2}$, Kaitlyn Braun ${ }^{1,2}$ and Donna M Wilcock ${ }^{1,2,6^{*}}$
}

\begin{abstract}
Background: Microglia are considered the resident immune cells of the central nervous system (CNS). In response to harmful stimuli, an inflammatory reaction ensues in which microglia are activated in a sequenced spectrum of pro- and antiinflammatory phenotypes that are akin to the well-characterized polarization states of peripheral macrophages. A "classically" activated M1 phenotype is known to eradicate toxicity. The transition to an "alternatively" activated M2 phenotype encompasses neuroprotection and repair. In recent years, inflammation has been considered an accompanying pathology in response to the accumulation of extracellular amyloid- $\beta$ (A $\beta$ ) in Alzheimer's disease (AD). This study aimed to drive an M2a-biased immune phenotype with IL-4 in vitro and in vivo and to determine the subsequent effects on microglial activation and $A \beta$ pathology.

Methods: In vitro, exogenous IL-4 was applied to BV2 microglial cell cultures to evaluate the temporal progression of microglial responses. In vivo, intracranial injections of an adeno-associate-virus (AAV) viral vector were performed to assess long-term expression of IL-4 in the frontal cortex and hippocampus of A $\beta$-depositing, APP/PS1 transgenic mice. Quantitative real-time PCR was used to assess the fold change in expression of biomarkers representing each of the microglial phenotypes in both the animal tissue and the BV2 cells. ELISAs quantified IL-4 expression and A 3 levels. Histological staining permitted quantification of microglial and astrocytic activity.
\end{abstract}

Results: Both in vitro and in vivo models showed an enhanced M2a phenotype, and the in vivo model revealed a trend toward a decreased trend in $A \beta$ deposition.

Conclusions: In summary, this study offers insight into the therapeutic potential of microglial immune response in AD. Keywords: Inflammation, Alzheimer's disease, Microglia, Beta-amyloid

\section{Introduction}

Alzheimer's disease (AD) was first identified in 1907 by Alois Alzheimer when investigating the case of a female patient, Auguste D, suffering from irrational personality changes and severe memory impairment. Post mortem analysis revealed a severely atrophied brain, and histological staining revealed amyloid- $\beta(A \beta)$ plaques and neurofibrillary

\footnotetext{
* Correspondence: donna.wilcock@uky.edu

'Department of Physiology, University of Kentucky, Sanders-Brown Center on Aging, Lexington, KY 40536, USA

2Department of Physiology, University of Kentucky, Lexington, KY 40536, USA Full list of author information is available at the end of the article
}

tangles composed of hyperphosphorylated and aggregated tau. In addition, Alzheimer noted a proliferated cluster of immune regulatory and support cells surrounding the $A \beta$ plaques and termed this "gliose", now known as the inflammatory response "gliosis" [1]. This observation signified a potential interaction between immune regulation and the development $A \beta$ lesions, a critical relationship that had previously been understudied until the last 2 decades [2]. Inflammation, the final pathology of the triad, marks the body's protective response to detrimental stimuli and injury. To date, an abundance of studies has confirmed the role of inflammation in the pathological progression of AD [3].

\section{Ciomed Central}

(C) 2015 Latta et al.; licensee BioMed Central. This is an Open Access article distributed under the terms of the Creative Commons Attribution License (http://creativecommons.org/licenses/by/4.0), which permits unrestricted use, distribution, and reproduction in any medium, provided the original work is properly credited. The Creative Commons Public Domain Dedication waiver (http://creativecommons.org/publicdomain/zero/1.0/) applies to the data made available in this article, unless otherwise stated. 
Derived from a mesodermal myeloid origin, microglia act in parallel to their sister immune regulatory cells of the periphery, macrophages, in the central nervous system (CNS) [4]. Current understanding of microglial physiology and activity, including phenotypic diversity, has been extrapolated. In response to immune stimulation, a spectrum of macrophage phenotypes is unveiled [5,6]. M1 activation, or classical activation, is considered the first line of defence to eradicate invading pathogens and is both driven and characterized by the release of proinflammatory cytokines such as interleukin $1 \beta$ (IL1 $\beta$ ) and tumor necrosis factor $\alpha$ (TNF $\alpha)$. On the other side of the spectrum, M2 activation, or alternative activation, is associated with wound repair and healing, as well as the ability to dampen the M1 response [7-10]. M2 activation is broken into additional sub-categories. M2a activation is characterized by the expression of mannose receptor MRC1 and extracellular matrix modeling ability with the expression of chitinase 3-like 3 proteins (YM1 in mice CHL3 in humans) and arginase 1, ARG1. Sequentially, acquired "deactivation" of the immune response is achieved by an M2c phenotype upon interleukin 10 (IL-10) exposure with amplified expression of transforming growth factor $\beta 1$ (TGF $\beta 1$ ) and sphingosine kinase 1 (SPHK1). The role of an M2b phenotype is relatively unknown. Nonetheless, it has been defined by an elevated expression of the CD86 and major histocompatibility complex II (MHCII) receptors in comparison to the low constitutive expression of these receptors across all activation phenotypes $[7,10]$.

An intricate cytokine network tightly controls the amplification and reduction of each of the microglial states. IL-4 is considered to be the strongest polarizing cytokine toward an M2a response [11]. IL-13 has also been noted as an M2a polarizing cytokine. The interaction of an M2a phenotype with $A \beta$ remains unclear. The induction of an M2a phenotype via IL-4 exposure has indicated both attenuation and amelioration of the neurotoxic peptide depositions in conflicting in vivo studies [12,13].

To assess M2a-driven modulation of microglial activation, IL-4 was endogenously applied to BV2 microglial cells and temporal changes in phenotype and morphology were monitored. Results revealed a robust M2a phenotype peaking at $8 \mathrm{~h}$ for the majority of phenotypic markers. Amplification of alternative phenotypic genes also revealed a peak in expression suggesting a minutely heterogeneous phenotype. Morphology showed reduced extended process upon IL-4 application, with the effects dampened over time and extended process formation. In order to better understand the effect of an M2a phenotype on A $\beta$ deposition, bilateral injections of an adeno-associated virus (AAV) expressing IL-4 were inserted into the frontal cortex and hippocampus of APP/PS1sw mice. A robust M2a phenotype was successfully induced with the AAV IL-4 injection. Histological staining revealing increased microgliosis and astrogliosis in the AAV-IL-4 injected mice. Lastly, A $\beta$ levels measured biochemically were moderately reduced in comparison to the mice treated with AAV-GFP, therefore providing insight into a potential mechanism of ameliorating $A \beta$ pathology in AD.

\section{Material and methods \\ BV2 cell culture}

BV2 cells were obtained from Dr. Linda Van Eldik, University of Kentucky, Lexington, KY. Cells were grown on six-well plates in FX12 media containing 10\% fetal bovine serum, $1 \%$ serum L-glutamate and $1 \%$ streptomycin (Life Technologies, Carlsbad, CA) until 80\% confluency was obtained approximately 3 days after passage. Cells were starved of serum for $24 \mathrm{~h}$ prior to treatment. Cells were stimulated with an exogenous application of murine IL-4 (20 ng/ml; R \& D Systems, Minneapolis, MN) in the serum-free medium added to the culture. Serum-free medium was applied as the no treatment control. Cells were incubated $\left(5 \% \mathrm{CO}_{2}, 37^{\circ} \mathrm{C}\right)$ for $2,4,6,8,10,16$ and $24 \mathrm{~h}$ before removal. Media were aspirated, and cells were rinsed twice in Dulbecco's phosphate-buffered saline $\mathrm{pH} 7.4$ (DPBS) (Life Technologies, Carlsbad, CA) at a $1 \times$ dilution for $5 \mathrm{~min}$ and frozen at $-80^{\circ} \mathrm{C}$. Cell culture experiments were performed in triplicate and repeated at least three times at different passage numbers.

\section{Immunofluorescent staining}

BV2 cells were grown on glass coverslips in 12-well plates until $80 \%$ confluency after approximately 3 days from passage. Twenty-four hours prior to the experiment, cells were starved and treated as above. After incubation cells were rinsed in $1 \times$ DPBS, transferred to a clean 12-well plate, fixed in 10\% neutral buffered formalin (Sigma-Aldrich, St Louis, MO) for $20 \mathrm{~min}$ and rinsed twice in $1 \times$ DPBS for $5 \mathrm{~min}$. Cells were blocked in 0.019\% L-lysine, 0.3\% triton-X (Sigma-Aldrich, St Louis, $\mathrm{MO}$ ) and $4 \%$ goat serum (Pel-Freez, Rogers, AR) in DPBS for $45 \mathrm{~min}$ and incubated in primary antibody CD11b 1:500 (Rat monoclonal, AbD Serotec, Raleigh, NC) in $1 \times$ DPBS overnight at $4^{\circ} \mathrm{C}$. Cells were left to acclimate to room temperature for $1 \mathrm{~h}$ and rinsed twice in $1 \times$ DPBS for $5 \mathrm{~min}$ before incubation in secondary goat anti-rat $(488 \mathrm{~nm})$ at a 1:20,000 dilution of for $1 \mathrm{~h}$. Glass coverslips were rinsed twice in $1 \times$ DPBS prior to adhesion to microscope slides using Permafluor mountant (Thermo Scientific, Fremont, CA). Fluorescence was visualized using Nikon Elements BR image analysis system (Melville, NY) at $60 \times$ magnification. The same settings were applied to all of the images.

\section{Animals}

This study was approved by the University of Kentucky Institutional Animal Care and Use Committee and 
conformed to the National Institutes of Health Guide for the Care and Use of Animals in Research. Fourteen APP/PS1 transgenic mice [14], a C57BL6 strain of mice with human APPSwe and PS1-dE9 mutations, were bred in the Division of Laboratory Animal Resources (DLAR) at the University of Kentucky and aged for 3 months prior to surgery. The animals were randomly assigned into one of two groups for adeno-associated virus type 8 (AAV-8) injection, either the AAV-GFP control group $(N=8)$ or the AAV-IL-4 group $(N=6)$. Data revealed no gender-dependent differences and were therefore analyzed together. Mice were killed 43 days post-surgery because of increased deaths in the IL-4-AAV-injected animals.

\section{AAV preparation}

The vector for preparing recombinant AAV was constructed by ligating the 1,349-bp EcoRI/SalI fragment carrying the IRES-GFP from pSMPUW-IRES-GFP (Cell Biolabs) into EcoRI/SalI-digested pZac2.1 (gift of Dr. Paul Murphy, University of Kentucky) to create ViCo1.28. The IL-4 insert was prepared by polymerase chain reaction (PCR) amplification of cDNA clones obtained from Open Biosystems (GE Healthcare, Dharmacon RNAi and gene expression, Piscataway, NJ). The IL-4 PCR primer sequence used was 'CCCGCTAGCG ACGGCACAGAGCTATTG.

CCACCGCGGGGCTCAGTACTACGAGTA' (Open Biosystems, GE Healthcare, Lafayette, CO; Cat. no.: MMM1013-7510313, clone ID 3376587 and accession $\mathrm{BC}$ 027514). The primers introduced a NheI site at the $5{ }^{\prime}$-end and a SacII site at the 3 '-end of each cytokine gene to facilitate cloning into the corresponding sites in ViCo1.28. The fidelity of each clone was confirmed by DNA sequence analysis.

AAV-8 coat protein-pseudotyped AAV-2 viruses were prepared by co-transfecting 10 T225 culture flasks of 293LTV cells (Cell Biolabs) with $250 \mathrm{mg}$ pAAV2/8 (obtained from the University of Pennsylvania Viral Core), $500 \mathrm{mg}$ pAdDF6 (gift of Dr. Paul Murphy, University of Kentucky) and, individually, $250 \mathrm{mg}$ of each cytokine clone using $5 \mathrm{mg}$ polyethyleneimine to enhance DNA uptake. After 3 days, the cells were harvested, washed, suspended in $13 \mathrm{ml}$ $150 \mathrm{mM} \mathrm{NaCl}, 50 \mathrm{mM}$ Tris $\cdot \mathrm{Cl} \mathrm{pH}$ 8.4, 0.5\% deoxycholate and $50 \mathrm{U} / \mathrm{ml}$ of benzonase and incubated at $37^{\circ} \mathrm{C}$ for $30 \mathrm{~min}$. An additional $2.8 \mathrm{ml} 5 \mathrm{M} \mathrm{NaCl}$ was added, and the incubation was continued for another $30 \mathrm{~min}$ at $45^{\circ} \mathrm{C}$. The cell suspension was then subjected to four freeze/thaw cycles $\left(30 \mathrm{~min}\right.$ at $-80^{\circ} \mathrm{C} / 30 \mathrm{~min}$ at $45^{\circ} \mathrm{C}$ ). The lysate was then partially clarified by centrifugation at $18,500 \times \mathrm{g}$ for $10 \mathrm{~min}$ at $20^{\circ} \mathrm{C}$. The supernatant was laid on top of an iodixanol step gradient and centrifuged at $350,000 \times \mathrm{g}$ for $1 \mathrm{~h}$ at $18^{\circ} \mathrm{C}$. The interface between the $40 \%$ and $54 \%$ iodixanol layers was withdrawn and spin-purified and concentrated using four washes with PBS in an Amicon Ultra-15 100,000 MWCO spin concentrator. The virus preparation was then titered using real-time PCR with primers directed against the CMV promoter region of the DNA encapsulated in the virions.

\section{Bilateral intracranial injections}

Mice were anesthetized with 5\% isoflurane (Butler-Schein, Dublin $\mathrm{OH}$ ) with oxygen levels kept at $1 \mathrm{~L}$ /minute and immobilized in stereotaxic apparatus. They were then maintained for the duration of surgery between $1 \%$ and $2 \%$ isoflurane. The skin was shaved and sterilized with a series of iodine and ethanol treatments before the cranium was exposed by a mid-sagittal incision. Four burr holes were made over the frontal cortex and hippocampus of both the left and right hemispheres using a dental drill mounted onto a stereotaxic frame. The coordinates for injection were confirmed by our laboratory prior to the study using a dye to visualize placement. Using bregma as the origin, burr holes were made in the frontal cortex $(+2.0 \mathrm{~mm}$ anteroposterior, $\pm 2.0 \mathrm{~mm}$ lateral $)$ and in the hippocampi $(-2.7 \mathrm{~mm}$ anteroposterior, $\pm 2.5 \mathrm{~mm}$ lateral) (as shown in Figure 1). At each of these sites, a 10- $\mu$ l Hamilton syringe fitted with a 26-guage needle was lowered to $-3.0 \mathrm{~mm}$ ventral to bregma (Hamilton, Reno, NV). Then $2 \mu \mathrm{l}$ of either AAV-GFP or AAV-IL-4 at a concentration of $5 \times 10^{10}$ genomes/ $\mu$ l was administered over a 2 -min period at each injection site. The mid-sagittal incision was cleaned and sutured, and a $4 \%$ lidocaine ointment was applied. Animals were group housed, based on sex, under standard vivarium conditions. Sutures were removed 10 days post-surgery.

\section{Tissue processing}

After 43 days post-surgery, the mice were administered a lethal intraperitoneal injection of $0.5 \mathrm{ml}$ of Buthanasia-D (Butler-Schein, Dublin $\mathrm{OH}$ ) and intracardially perfused with $25 \mathrm{ml} \mathrm{0.9 \%}$ saline (Ricca Chemical Co., Arlington, TX). The brain was removed immediately and bisected along the mid-sagittal plane. The left hemisphere was post-fixed in $4 \%$ paraformaldehyde for $24 \mathrm{~h}$ before being treated for cyroprotection through a series of 10, 20 and 30\% sucrose concentrations each for an additional $24 \mathrm{~h}$. The left hemisphere was placed on a frozen stage and sectioned into $25-\mu \mathrm{m}$ horizontal sections, which were collected using a sliding microtome. The sections were stored free-floating in $1 \times$ DPBS plus sodium azide at $4^{\circ} \mathrm{C}$. Sections were stored in 24-well plates at $4{ }^{\circ} \mathrm{C}$ until required. The frontal cortex and hippocampus of the right hemisphere were micro-dissected, snap-frozen in liquid nitrogen and stowed at $-80^{\circ} \mathrm{C}$.

\section{Immunohistochemistry}

A Nissl stain was used to determine the injection site location determined as previously described [15]. Eight 

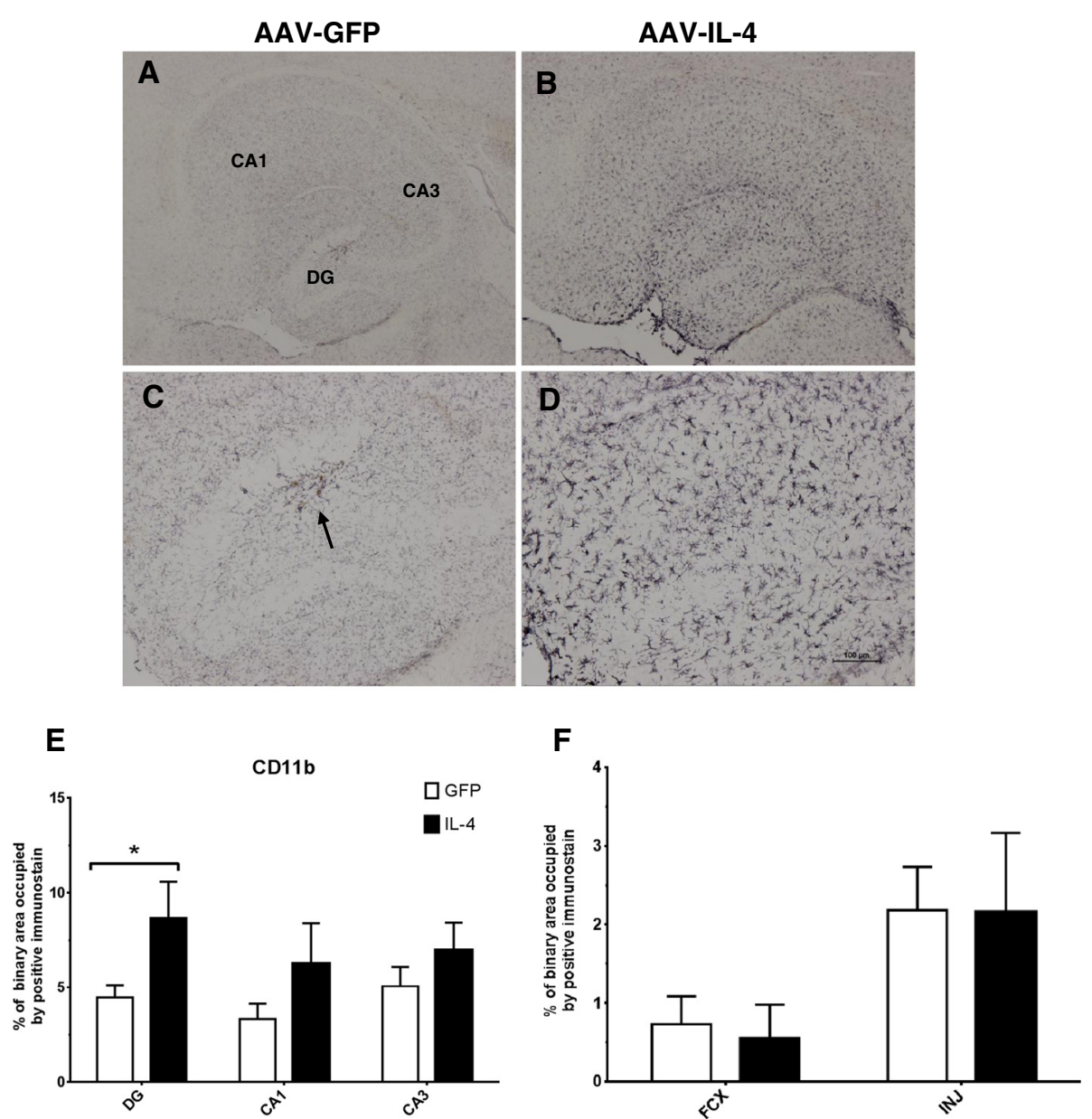

Figure 1 IL-4 shows increased CD11b immunostaining. A and $\mathbf{B}$ CD11b staining of hippocampal sections of the AAV-GFP- and the AAV-IL-4-injected mice, respectively. $\mathbf{C}$ and $\mathbf{D}$ Staining in the dentate gyrus (DG); an arrow shows the site of the hippocampal injection. $\mathbf{E}$ The percentage of binary area occupied by the positive immunostain in the hippocampus across all samples; $\mathbf{F}$ uses $(N=4)$ for AAV-IL4 samples because of damage in the frontal cortex of two AAV-IL-4 mice. ${ }^{*} P<0.05$ between the AAV-GFP control $(N=8)$ and AAV-IL-4 $(N=6)$.

free-floating $25-\mu \mathrm{m}$ sections spaced $\sim 400 \mu \mathrm{m}$ from each other were collected $1,300-3,400 \mu \mathrm{m}$ ventral to bregma and separated into $1 \times$ DPBS. Initially, the free floating sections were permeabilized, and endogenous peroxidase sites were inhibited via incubation with $10 \%$ methanol and $3 \%$ hydrogen peroxide in $1 \times$ DPBS for $15 \mathrm{~min}$. Sections were blocked with $0.3 \%$ triton-X, $4 \%$ goat serum and $0.019 \%$ L-lysine in $1 \times$ DPBS. Sections were incubated in primary antibodies CD11b, 1:3,000 (Rat monoclonal, AbD Serotec, Raleigh, NC) and GFAP, 1:10,000, (Rabbit polyclonal, Dako, Denmark) with $4 \%$ goat serum in DPBS left at room temperature and then overnight at $4^{\circ} \mathrm{C}$. After incubation and three 5-min washes in 1× DPBS, sections were incubated in biotinylated secondary antibodies; goat anti-rabbit IgG was used for GFAP and goat anti-rat for CD11b, both at 1:3,000 (Vector Laboratories, Burlingame, CA) for $2 \mathrm{~h}$. Amplification of the secondary antibody signal was achieved through incubation in advidin-biotin complex (ABC) (Vector Laboratories, Burlingame, CA) for $1 \mathrm{~h}$. For color development the vector diaminobenzidine (DAB) peroxidase kit (Vector Laboratories, Burlingame, CA) was used according to the manufacturer's instructions. Sections were mounted onto slides, left to air-dry overnight, dehydrated in an ethanol gradient followed by xylene incubations and cover-slipped using DPX mountant (Electron Microscopy Sciences, Hatfield, PA).

Images of the stained sections were taken at 200x magnification using the Nikon Elements BR image analysis system (Melville, NY) in the frontal cortex (FCX). Hippocampal fields were localized to the dentate gyrus (DG), cornu ammonis 1 (CA1) and cornu ammonis 3 (CA3) and images collected at the same magnification. Anatomical structures in the tissue were used to ensure uniformity in the image. Microscope settings were saved and were the same for all of the images in each stain. A threshold for positive immunostaining was set, saved and applied to all of the images of a given stain. The data yielded the percentage of binary area occupied by the 
positive immunostain. Approximately six sections per mouse were used for analysis in the hippocampus and the frontal cortex. Tissue damage in the frontal cortex of several animals left large holes interfering with quantification of the positive immunostain analysis. Therefore, two mice in the AAV-IL-4 group were exempt from this analysis.

\section{ELISA measurements}

Protein was extracted from the frontal cortex tissue by homogenization in mammalian protein extraction reagent (MPER) lysis buffer with protease and phosphatase inhibitor (Pierce Biotechnology Inc., Rockford, IL). The samples were centrifuged for $15 \mathrm{~min}$ at $10,000 \mathrm{rg}$ at $4^{\circ} \mathrm{C}$. The supernatant was collected as the soluble extract, leaving a pellet of remaining insoluble extract. A Bicinchonic Acid protein assay kit (BCA) (Pierce Biotechnology Inc., Rockford, IL) was performed to obtain the concentration of the soluble extract according to the manufacturer's instructions. The soluble extracts were normalized to the lowest concentration using the MPER lysis buffer. Then $250 \mu \mathrm{l}$ of $70 \%$ formic acid was added to the insoluble extract before ultracentrifugation at $47,000 \mathrm{rpm}$ at $4^{\circ} \mathrm{C}$ for $1 \mathrm{~h}$. The supernatant was collected and neutralized 1:20 with Tris- $\mathrm{HCl}(\mathrm{pH} 11)$. Additional $\mathrm{HCl}$ was added to bring the sample accurately to $\mathrm{pH} 7$ using a drop pipet. A Meso Scale Discovery (MSD) 96-Well MULTI-SPOT Human (6E10) A-beta Triplex Assay (MSD, Gaithersburg, MD) was performed on both the soluble and insoluble extractions, according to the manufacturer's instructions, to determine the concentrations of $\mathrm{A} \beta 38, \mathrm{~A} \beta 40$ and $\mathrm{A} \beta 42$. Murine IL-4 levels in the soluble extracts were detected using the the Mouse IL-4 Quantikine ELISA kit (R \& D Systems Inc., Minneapolis, MN). All steps followed the manufacturer's instructions. A second BCA was performed on soluble extracts to confirm the protein concentration, and samples were normalized prior to the ELISA measurement.

\section{Quantitative real-time RT-PCR}

RNA was extracted from the frozen hippocampus of the right hemisphere by rotor homogenization Bio-Gen Pro200 (Pro-Scientific, Oxford, CT) in the RNA-Solv Reagent of the RNeasy tissue kit (Qiagen, Valencia, CA); the subsequent steps of the extraction were achieved according to the manufacturer's instructions. BV2 cell homogenization RNA was extracted from the BV2 cells using cell scrapers in RLT buffer of the RNeasy Mini-kit (Qiagen, Valencia, CA). Again, the subsequent steps of the extraction were achieved according to the manufacturer's instructions. The Biospec-Nano Spectrophotometer (Shimadzu, Kyoto, Japan) was used to quantify the nucleic acid concentration in the RNA samples. The RNA concentrations were standardized to the lowest concentration with RNAase free water. The standardized samples were added to the High Capacity RNA-to-cDNA kit (Applied Biosystems, Foster City, CA) including reverse transcriptase, random primers and buffer according to the manufacturer's instructions. The cDNA was produced through a series of heating and annealing cycles in the Veriti $^{\text {Ti }}$ 96-well Thermocycler (Applied Biosystems, Grand Island, NY).

Quantitative real-time RT-PCR was achieved using the Fast-TaqMan Gene Expression Kit in 96-well plates (Life Technologies, Carlsbad, CA); $0.5 \mu \mathrm{l}$ of the cDNA (100 $\mu \mathrm{l}$ of standardized RNA samples) was diluted with $6.5 \mu \mathrm{l}$ of RNAse-free water and added to each well; $1 \mu$ of the selected gene probe (Life Technologies, Carlsbad, CA) listed in Table 1 was added to $10 \mu \mathrm{l}$ of the Fast-TaqMan reagent and added to each well. Real-time RT-PCR was performed using the $\mathrm{ViiA}^{\mathrm{w}} 7$ Real Time PCR system (Applied Biosystems, Grand Island, NY). All genes measured were normalized to the constitutively expressed $18 \mathrm{~S}$ rRNA gene. The gene expression of the AAV-IL-4 mice was compared relative to the AAV-GFP control group. Fold change was calculated using the $2^{-\Delta \Delta \mathrm{Ct}}$ method [16].

\section{Data analysis}

For the in vitro analysis, normalized fold change values were log-transformed in order to apply parametric statistical methods. Repeated measures ANOVA, with an unstructured covariance matrix, was then used to estimate mean log fold change at each time point within each gene. The time point at which expression peaked was determined by post hoc testing such that the largest point estimate observed was then compared to the

Table 1 Genes for RT-qPCR

\begin{tabular}{lll}
\hline Gene of interest & PMID & Taqman ID \\
\hline IL-1 $\beta$ & NM_008361.3 & Mm00434228_m1 \\
IL-12 & NM_008351.2 & Mm00434165_m1 \\
TNFa & NM_013693.3 & Mm00443258_m1 \\
IL-6 & NM_031168.1 & Mm00446190_m1 \\
IFNY & NM_008337.3 & Mm01168134_m1 \\
IL1ra & NM_031167.5 & Mm00446186_m1 \\
ARG1 & NM_007482.3 & Mm00475988_m1 \\
YM1 (Chil3) & NM_009892.2 & Mm00657889_mH \\
FIZZ1 & NM_020509.3 & Mm00445109_m1 \\
MRC1 & NM_008625.2 & Mm00485148_m1 \\
CD86 & NM_019388.3 & Mm00444543_m1 \\
FCYR1 & NM_010186.5 & Mm00438874_m1 \\
FCYR3 & NM_010188.5 & Mm00438882_m1 \\
TGF 11 & NM_011577.1 & Mm01178820_m1 \\
SPHK1 & NM_011451.3 & Mm01252544_m1 \\
\hline
\end{tabular}




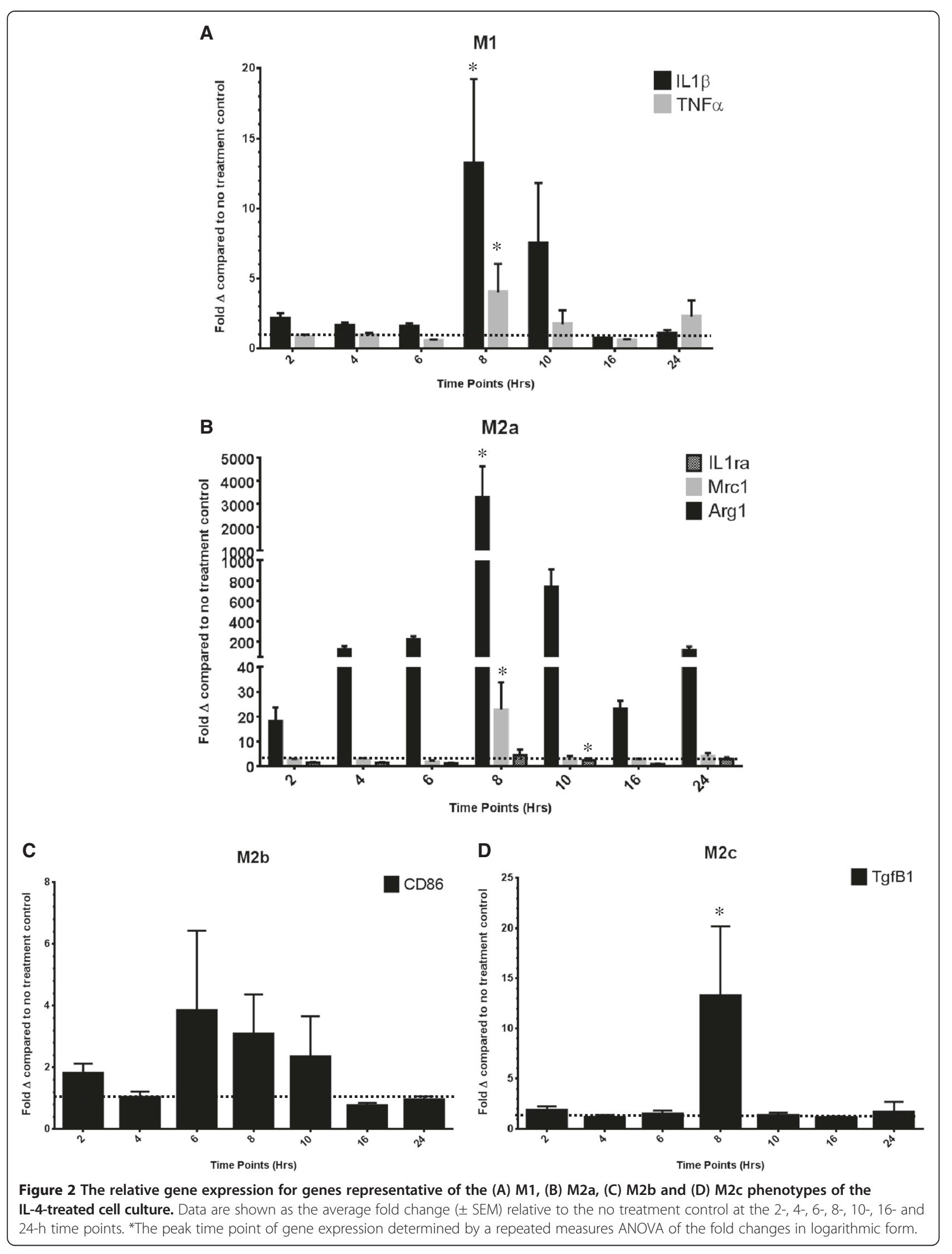


Table $2 \boldsymbol{P}$ values for individual time-point comparisons for qRT-PCR in IL-4-treated BV2 cell culture

\begin{tabular}{|c|c|c|c|c|c|c|c|c|}
\hline IL1 $\beta$ & & 2 & 4 & 6 & 8 & 10 & 16 & 24 \\
\hline & 2 & & 0.9613 & 0.3623 & 0.0024 & 0.3746 & 0.0354 & 0.0218 \\
\hline & 4 & & & 0.4365 & 0.0270 & 0.2481 & 0.0405 & 0.168 \\
\hline & 6 & & & & 0.0122 & 0.2677 & 0.0698 & 0.1067 \\
\hline & 8 & & & & & 0.4261 & 0.0024 & 0.0006 \\
\hline & 10 & & & & & & 0.0792 & 0.0520 \\
\hline & 16 & & & & & & & 0.8212 \\
\hline & 24 & & & & & & & \\
\hline \multirow[t]{8}{*}{ TNFa } & & 2 & 4 & 6 & 8 & 10 & 16 & 24 \\
\hline & 2 & & 0.2953 & 0.0987 & 0.0252 & 0.9023 & 0.6955 & 0.5087 \\
\hline & 4 & & & 0.0095 & 0.4168 & 0.5882 & 0.2381 & 0.9796 \\
\hline & 6 & & & & 0.0372 & 0.3117 & 0.1469 & 0.0680 \\
\hline & 8 & & & & & 0.3731 & 0.1441 & 0.5100 \\
\hline & 10 & & & & & & 0.7412 & 0.7478 \\
\hline & 16 & & & & & & & 0.4097 \\
\hline & 24 & & & & & & & \\
\hline \multirow[t]{8}{*}{ IL1ra } & & 2 & 4 & 6 & 8 & 10 & 16 & 24 \\
\hline & 2 & & 0.8337 & 0.2886 & 0.9078 & 0.6454 & 0.2457 & 0.6626 \\
\hline & 4 & & & 0.2485 & 0.8572 & 0.6258 & 0.2486 & 0.7000 \\
\hline & 6 & & & & 0.7178 & 0.4069 & 0.6078 & 0.2248 \\
\hline & 8 & & & & & 0.6661 & 0.5646 & 0.7672 \\
\hline & 10 & & & & & & 0.3647 & 0.8656 \\
\hline & 16 & & & & & & & 0.2865 \\
\hline & 24 & & & & & & & \\
\hline \multirow[t]{8}{*}{ MRC1 } & & 2 & 4 & 6 & 8 & 10 & 16 & 24 \\
\hline & 2 & & 0.7488 & 0.3842 & 0.0007 & 0.5648 & 0.5007 & 0.8252 \\
\hline & 4 & & & 0.1018 & 0.0025 & 0.4226 & 0.3949 & 0.5433 \\
\hline & 6 & & & & 0.0048 & 0.8499 & 0.8391 & 0.5605 \\
\hline & 8 & & & & & 0.0003 & 0.0205 & 0.0083 \\
\hline & 10 & & & & & & 0.7647 & 0.7846 \\
\hline & 16 & & & & & & & 0.6493 \\
\hline & 24 & & & & & & & \\
\hline \multirow[t]{8}{*}{ ARG1 } & & 2 & 4 & 6 & 8 & 10 & 16 & 24 \\
\hline & 2 & & 0.0138 & $<.0001$ & $<.0001$ & 0.0004 & 0.0208 & 0.0085 \\
\hline & 4 & & & 0.0106 & 0.0020 & 0.0041 & 0.4579 & 0.7186 \\
\hline & 6 & & & & 0.0018 & 0.2438 & $<.0001$ & 0.0012 \\
\hline & 8 & & & & & 0.1388 & $<.0001$ & $<.0001$ \\
\hline & 10 & & & & & & 0.0014 & 0.0156 \\
\hline & 16 & & & & & & & 0.1544 \\
\hline & 24 & & & & & & & \\
\hline \multirow[t]{4}{*}{ CD86 } & & 2 & 4 & 6 & 8 & 10 & 16 & 24 \\
\hline & 2 & & 0.2042 & 0.4318 & 0.9169 & 0.5731 & 0.2545 & 0.0203 \\
\hline & 4 & & & 0.8782 & 0.3780 & 0.8207 & 0.8871 & 0.4193 \\
\hline & 6 & & & & 0.5538 & 0.9294 & 0.8339 & 0.5795 \\
\hline
\end{tabular}

Table $2 \boldsymbol{P}$ values for individual time-point comparisons for qRT-PCR in IL-4-treated BV2 cell culture (Continued)

\begin{tabular}{|c|c|c|c|c|c|c|c|}
\hline & 8 & & & & 0.6455 & 0.4060 & 0.1343 \\
\hline & 10 & & & & & 0.8108 & 0.6164 \\
\hline & 16 & & & & & & 0.7600 \\
\hline & 24 & & & & & & \\
\hline TGF $\beta 1$ & 2 & 24 & 6 & 8 & 10 & 16 & 24 \\
\hline & 2 & 0.7553 & 0.8404 & 0.0200 & 0.8186 & 0.7694 & 0.2791 \\
\hline & 4 & & 0.6795 & 0.1164 & 0.9889 & 0.8582 & 0.0118 \\
\hline & 6 & & & 0.0406 & 0.8702 & 0.9094 & 0.0356 \\
\hline & 8 & & & & 0.2138 & 0.0431 & 0.0127 \\
\hline & 10 & & & & & 0.9236 & 0.1373 \\
\hline & 16 & & & & & & 0.0771 \\
\hline & 24 & & & & & & \\
\hline
\end{tabular}

remaining time points. No ties were observed for peak time (i.e., the largest point estimate was identifiable for each gene). Repeated measures analyses were performed using the MIXED procedure in SAS 9.3 ${ }^{\circ}$ (SAS Institute, Inc., Cary, NC). Otherwise, group differences were assessed using two-sample Student's $t$-tests. Analysis tests and data representation were performed using GraphPad Prism version 6.01 for Windows (GraphPad Software, La Jolla, CA, USA). Statistical significance was set at 0.05 .

\section{Results}

To determine the effect of the murine IL-4 application on BV2 cells, RNA was extracted, and qRT-PCR was performed on the gene markers specific to each phenotype (M1, M2a, M2b, M2c) as shown in Figure 2. Fold change was calculated relative to cell culture exposed to a no-treatment control. Both IL1 $\beta$ and TNF $\alpha$ of the M1 phenotype show a peak at $8 \mathrm{~h}$ (Figure 2A). In the M2a phenotype, ARG1 is largely amplified to 3,500 fold at $8 \mathrm{~h}$; additionally, MRC1 also peaks at $8 \mathrm{~h}$. IL1ra shows a significant peak at $10 \mathrm{~h}$ post treatment (Figure 2B). YM1 was found to have aberrant expression in both the control and IL-4-treated cells and was therefore exempt from the analysis. No significant differences can be seen in the M2b phenotype over time (Figure 2C), but we found elevated levels of Tgf $\beta 1$ in the M2c phenotype (Figure 2D). Table 2 shows the individual $P$ values for the comparisons of gene expression over the time course.

The morphology of the CD11b-positive BV2 cells over this time course was monitored by immunofluorescent staining, shown in Figure 3. Generally, CD11b-positive microglial cells did not differ after treatment with IL-4 in spite of a clear difference in the inflammatory phenotype. We did observe a qualitatively decreased density of extended processes of the BV2 cells in the IL-4-treated 

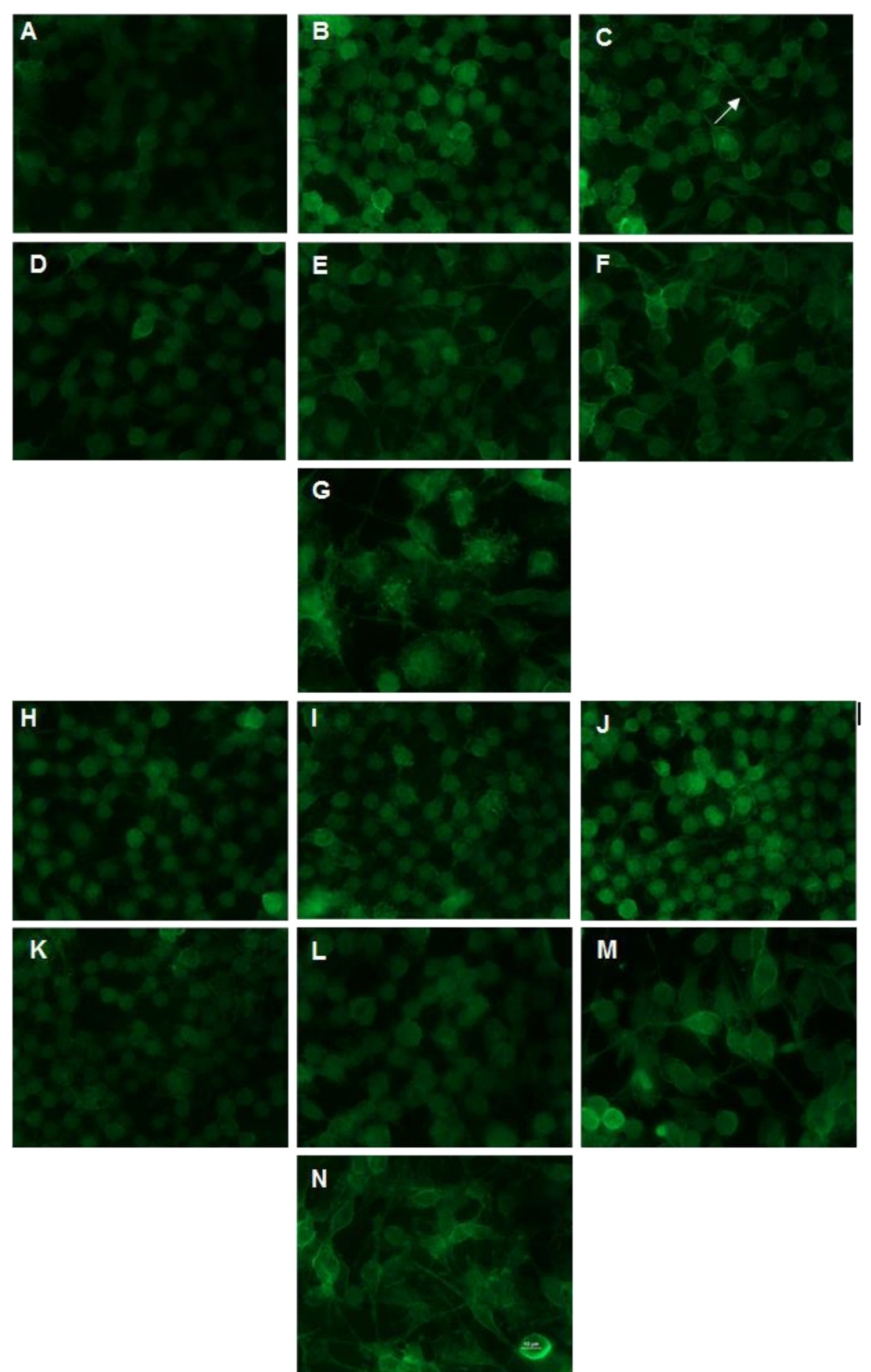

Figure 3 BV2 cell culture exposed to CD11b immunofluorescent staining over time. A-G Immunofluorescent staining of no-treatment control cells at 2, 4, $68,10,16$ and 24 h, respectively. H-N Staining of cells exposed to the IL-4 treatment at 2, 4, 6, 8 10, 16 and 24 h, respectively. Images were taken with approximately the same cell confluency at a magnification of $60 \mathrm{x}$ and show a higher density of processes in the no treatment control compared to the IL-4-treated cells until 16 h. Arrow indicates extended processes seen on the untreated BV2 cells at $6 \mathrm{~h}$. 
groups compared to the no-treatment control groups, but this was not quantifiable (Figure 3 ).

A sandwich ELISA was performed to confirm that the AAV-8 was an effective vehicle for gene delivery resulting in IL-4 expression. Protein extracts taken from the right frontal cortex showed a marked significant increase $(P=0.0145)$ in $\mathrm{IL}-4$ levels (mean $32.2 \mathrm{ng} \mathrm{IL}-4 / \mathrm{mg}$ protein) in the mice receiving AAV-IL-4 in comparison to the AAV-GFP-treated mice (mean $2.4 \mathrm{ng}$ IL-4/mg protein). Figure 4 indicates the neuroinflammatory profile of the AAV-IL-4 mice relative to that of the AAV-GFP controls. Real-time $\mathrm{qPCR}$ was performed from RNA extracts from the right hippocampus. Genetic probes were selected based on their role in M1, M2a, M2b and M2c phenotypes identified in peripheral macrophages. As hypothesized, a robust M2a phenotype can be observed as a result of the AAV-IL-4 injection. IL1ra, YM1, ARG1 and FIZZ1 proved to be significantly different from the control. In particular, YM1 and FIZZ1 showed a 700-1,000-fold change. Elevated expression of M1-specific genes can also be identified with significant increases in IL1 $\beta$ and IL12a.

To determine the morphology and activation of microglia in response to the AAV-IL-4 treatment, tissue sections were stained with a $\beta$-integrin marker, CD11b. In the hippocampus, Figure $1 \mathrm{~A}$ and $\mathrm{B}$ shows an increased trend in positive CD11b immunostaining. In particular, the DG reveals a positive increase in the positive immunostaining (Figure $1 \mathrm{C}$ and D). An arrow in Figure $1 \mathrm{C}$ highlights microglial activation around the injection site of the AAV-IL-4 mice. Interestingly, we found increased CD11b staining in the hippocampus of AAV-IL-4

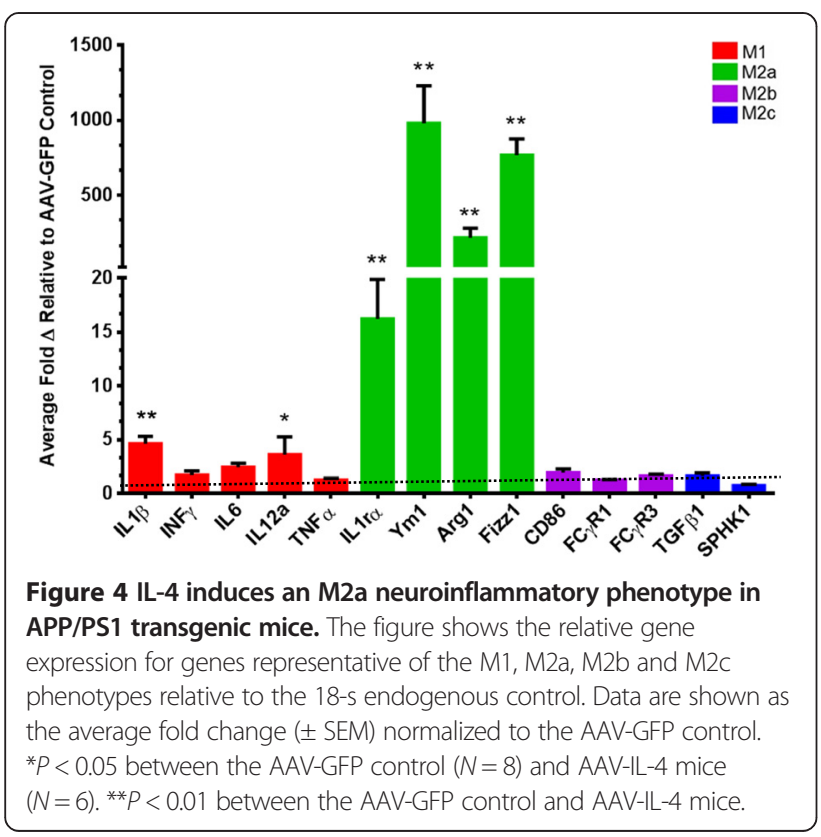

mice, which was statistically significant in the dentate gyrus region (Figure 1E), but we did not see changes in the frontal cortex (Figure 1F).

In addition, immunostaining was conducted with GFAP to assess the effect of the AAV-IL-4 treatment on astrogliosis. Figure $5 \mathrm{~A}$ and $\mathrm{B}$ shows a modest increase in positive immunostaining, exemplified in Figure $5 \mathrm{C}$, but fails to show any significant results. Once again, analysis on the frontal cortex was performed on a sample size of $N=4$ in the AAV-IL- 4 mice, consistent to that of the CD11b stain. It should be noted that an increase in astroglial staining could be observed in the AAV-GFP mice around the hippocampal injection site.

$\mathrm{A} \beta$ depositions in the APP/PS1 transgenic mice were determined biochemically on the MPER soluble and insoluble (formic acid soluble) protein extracts. An MSD ELISA multiplexed assay was used to analyze the concentration of $A \beta 1-38, A \beta \quad 1-40$ and $A \beta \quad 1-42$ in the $\mathrm{pg} / \mathrm{mg}$ of the protein extract (Table 3). Modest decreases are shown in both the $A \beta$ soluble extracts despite no marked significance (Figure 6A). An exception to this is the $A \beta 1-42$ depositions in the insoluble extract. Immunostaining for $A \beta$ and Congo red staining proved to be inconclusive because of the scarce compact plaque formation due to the young age of the survival of the mice. Values for the $A \beta$ species concentrations are given in Table 4.

\section{Discussion}

Inflammation and $\mathrm{A} \beta$ are suggested to work both independently and synergistically, contributing to the development of AD symptoms and pathological progression [2,17-19]. It remains unknown whether inflammation has a direct or indirect effect on the acceleration of $A \beta$ pathology, with data showing both increased and decreased amyloid deposition [20,21]. The current study aimed to characterize the M2a response stimulated by IL-4 both in vitro and in vivo. Further, we investigated the effect of an M2a phenotype on $A \beta$ levels in the brain. The time courses of microglial morphology and phenotypic alterations in response to the application of IL-4 applied exogenously were assessed using the immortalized murine microglial cell line, BV2. In vivo, IL-4 was delivered to the brain using an AAV vector in the APP/PS1 transgenic mouse model. IL-4 is a typical inducer of an M2a phenotype [11]. An M2a phenotype was indeed induced following IL-4 application using both in vitro and in vivo approaches. The BV2 microglial cells showed a time-dependent heterogeneous phenotype with the M2a response being the most robust. These results concurred with the in vivo study; again, an evident M2a phenotype was induced with depleted $A \beta$ deposits. Unfortunately, due to significant 

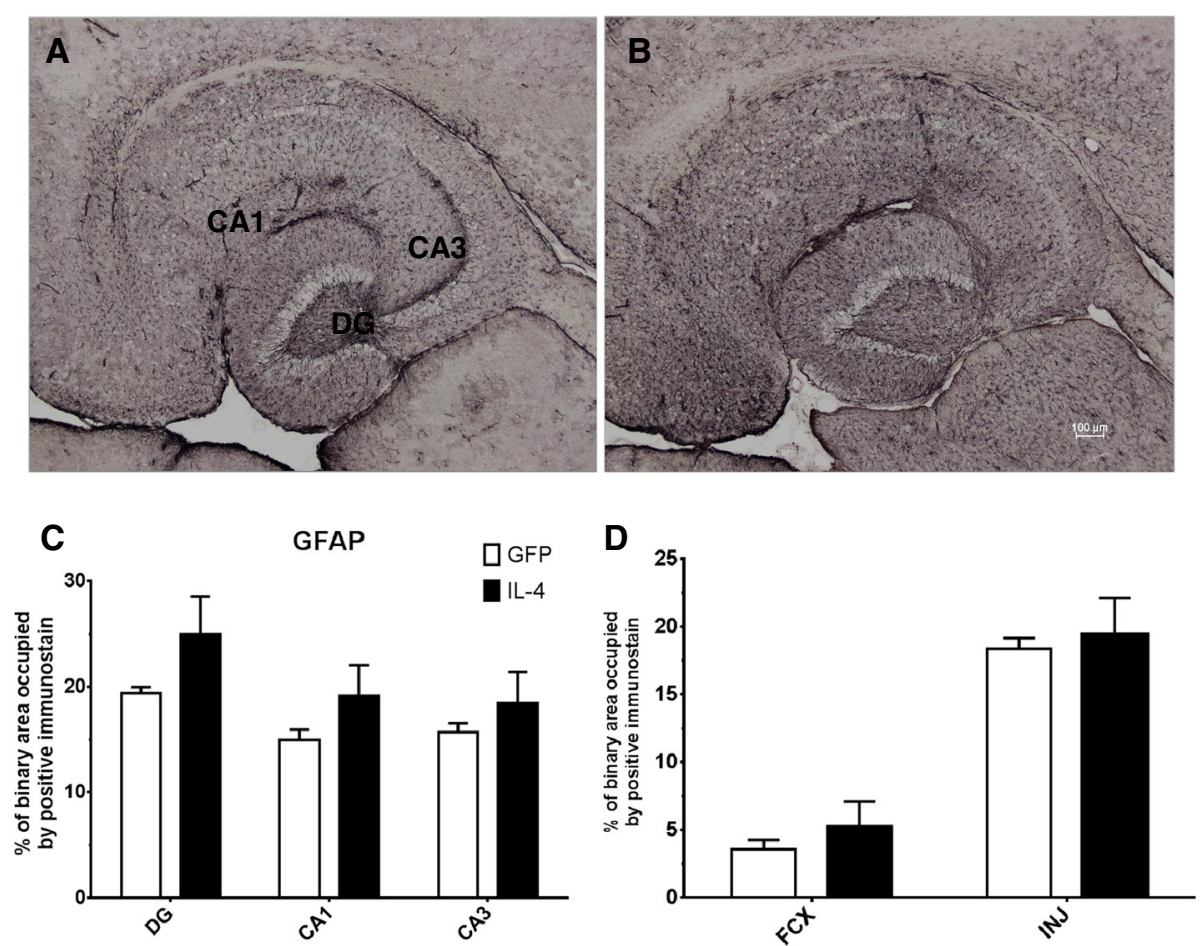

Figure 5 IL-4 shows increased GFAP immunostaining. A and B GFAP staining of hippocampal sections of the AAV-GFP- and the AAV-IL-4-injected mice, respectively. C The percentage of binary area occupied by the positive immunostain in the hippocampus across all samples; $\mathbf{D}$ uses $(N=4)$ for AAV-IL4 samples because of damage in the frontal cortex of two AAV-IL-4 mice. ${ }^{*} P<0.05$ between the AAV-GFP control $(N=8)$ and AAV-IL-4 (N=6).

death in the mice receiving the IL-4 AAV, we terminated the study prematurely; thus, our measurement of $A \beta$ deposition was restricted to biochemical outcomes.

IL-4 was selected as our stimulus for an M2a phenotype as it has been shown to be the standard stimulator of an M2a phenotype in peripheral macrophages [11]. Application of IL-4 onto BV2 cells revealed a robust time-dependent increase in M2a phenotypic markers with the vast majority showing a statistically significant peak in expression at $8 \mathrm{~h}$. Overall, phenotypic expression in IL-4-induced BV2 cells strongly validates the gene screening seen in the in vivo model. In addition to the exhibition of a robust M2a state, increases in M1 and M2c could also be observed indicating an overall heterogeneous phenotype in response to the IL-4 application. This heterogeneity suggests links between these markers, emphasizing the spectral nature of microglial phenotypes and the potential interplay of the mechanistic actions of downstream pathways. Hence, further investigations are required to decipher these cascades. The time course shows that IL1 $\beta$ is increased in the BV2 cells in direct proportion to the increase in the IL1ra. It is possible that the increase in IL1ra is a homeostatic response to counteract amplified expression of IL1 $\beta$. IL1 $\beta$ has a diverse range of physiological roles within the CNS [22]; therefore the benefits of complete antagonism to achieve an M1 phenotype could be detrimental to maintaining overall homeostasis within the tissue. In addition, the M2a phenotypic genes ARG1 and MRC1 both peak at $8 \mathrm{~h}$. Future studies may examine other mechanisms by which we can promote an M2a response. For example, IL-13 is also an M2a-promoting cytokine, overlapping with the IL-4 pathway by dimerization of the shared IL-4R $\alpha$ with IL-13R $\alpha 1$ to activate a STAT-6 mechanism [23].

Immunofluorescent $\mathrm{CD} 11 \mathrm{~b}$ was used to visualize the microglial morphology at the sequential time points.

Table 3 Soluble and insoluble $A \beta$ levels measured by ELISA

\begin{tabular}{|c|c|c|c|c|c|c|}
\hline \multirow[t]{2}{*}{ AAV injection } & \multicolumn{3}{|c|}{ Soluble (pg/ug protein) } & \multicolumn{3}{|c|}{ Insoluble (ng/ug protein) } \\
\hline & $A \beta-38$ & $A \beta-40$ & $A \beta-42$ & $A \beta-38$ & $A \beta-40$ & $A \beta-42$ \\
\hline GFP-AAV & $30.6 \pm 12.6$ & $80.4 \pm 17.7$ & $105.6 \pm 26.8$ & $204.6 \pm 82.1$ & $191.5 \pm 62.6$ & $461.8 \pm 96.0$ \\
\hline IL-4-AAV & $13.2 \pm 6.7$ & $60.4 \pm 16.3$ & $45.2 \pm 8.6$ & $83.0 \pm 45.1$ & $62.6 \pm 12.1$ & $472.2 \pm 181.5$ \\
\hline
\end{tabular}

Bold font indicates $P<0.05$ compared to GFP-AAV of the same time point. 

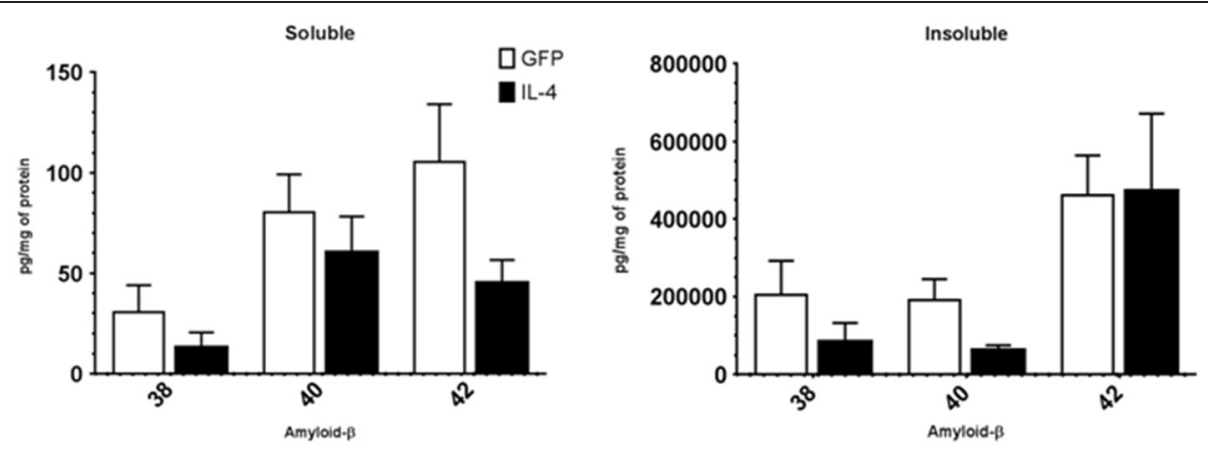

Figure $\mathbf{6}$ IL-4 causes a small decrease in $\mathbf{A} \boldsymbol{\beta}$ deposition. This figure shows the relative amyloid- $\beta$ expression $(1-38,1-40,1-42)$ detected using a Meso Scale Discovery assay (MSD) for the AAV-GFP control and the AAV-IL-4-treated APP/PS1 transgenic mice. No statistically significant difference was seen between the AAV-GFP control and AAV-IL-4 groups, but the results show a decreasing trend ( $N=8$ AAV-GFP and $N=6$ for AAV-IL-4). Immunostained $A \beta$ plaques with Congo red could not be detected because the mice were only 3 months old. Compact plaques can be seen at 6 months of age.

CD11b is a microglial surface integrin expressed solely during activation, and it was previously indicated that disruption to homeostasis induces an "amoeboid" retraction of ramified processes, an enlarged cytoplasm, which is an indication of the activated state [24,25]. Extended filopedia can be seen up until the 6-h time point in the no-treatment control, suggesting a maintained resting state as opposed to the retracted and condensed shape compared to that of the IL-4-treated cells demonstrating activation. There is no distinct correlation between the morphology at the time points with the inflammatory profile despite the minor morphological changes discussed.

Investigation of the inflammatory profile from RT-qPCR of the right hippocampus of mice injected with IL-4

Table 4 Fold change values for RT-PCR in APP/PS1 transgenic mice

\begin{tabular}{|c|c|c|}
\hline Gene & GFP-AAV & IL-4-AAV \\
\hline IL1 $\beta$ & $1.20 \pm 0.27$ & $4.63 \pm 0.67$ \\
\hline INFY & $1.20 \pm 0.39$ & $1.70 \pm 0.32$ \\
\hline IL6 & $1.33 \pm 0.40$ & $2.43 \pm 0.38$ \\
\hline IL12a & $1.18 \pm 0.31$ & $3.60 \pm 1.64$ \\
\hline TNFa & $0.74 \pm 0.51$ & $1.24 \pm 0.15$ \\
\hline IL1ra & $1.41 \pm 0.44$ & $16.24 \pm 3.62$ \\
\hline YM1 & $1.90 \pm 1.04$ & $979.43 \pm 251.30$ \\
\hline ARG1 & $1.27 \pm 0.40$ & $216.17 \pm 65.91$ \\
\hline $\mathrm{FIZZ1}$ & $2.80 \pm 1.74$ & $767.81 \pm 108.26$ \\
\hline CD86 & $1.19 \pm 0.29$ & $1.91 \pm 0.36$ \\
\hline $\mathrm{FC} \gamma \mathrm{R} 1$ & $1.22 \pm 0.33$ & $1.23 \pm 0.07$ \\
\hline $\mathrm{FC} \gamma \mathrm{R} 3$ & $1.15 \pm 0.26$ & $1.61 \pm 0.17$ \\
\hline TGF $\beta 1$ & $1.21 \pm 0.30$ & $1.62 \pm 0.28$ \\
\hline SPHK1 & $1.03 \pm 0.10$ & $1.61 \pm 0.12$ \\
\hline
\end{tabular}

Bold font indicates $P<0.05$ compared to GFP-AAV of that time point and genotype. expressing AAV indicated a robust M2a phenotype with significant elevations in all inflammatory markers. Extracellular matrix remodeling proteins YM1 and FIZZ1 showed highly amplified levels reaching 500-1,000 fold. IL1ra showed a marked increase, neutralizing the role of IL1 $\beta$. Congruent to the trend identified in the BV2 cells, the immune profile also exhibited increases in M1 phenotypic genes, specifically IL1 $\beta$ and IL-12a, suggesting a heterogeneous immune profile with chronic exposure to IL-4. Furthermore, this exemplifies the continuum of functionality in which microglia lie. It is evident that elevation of the M1 phenotype has responsive ties to that of the M2a phenotype, as seen in the BV2 cells. Phenotypic heterogeneity was further enforced by Weekman et al. [26], who revealed a mixed phenotype with IFN $\gamma$ stimulation after 6 months of exposure. Unlike the BV2 cells, elevations of an M2b or M2c could not be observed in the mouse tissue.

CD11b immunohistochemistry is used to visualize activated microglia generating an indication of gliosis in a given tissue area. Astrocytes are also considered to have an immune-regulatory role. GFAP staining visualizes astrogliosis, proliferation of astrocytes in a given area. In this study, the data reveal increases in positive immunostaining in response to the AAV-IL-4 treatment, indicating a responsive proliferation of both cell subsets associated with elevated M2a phenotypic expression. Specifically, staining with $\mathrm{CD} 11 \mathrm{~b}$ revealed marked, statistically significant positive immunostaining in the dentate gyrus of the hippocampus, suggesting increased microgliosis in this region. Data from the GFAP staining also show increased immunoreactivity, but there was no statistical significance in any region of the hippocampus or frontal cortex. What is particularly intriguing is that these markers of "inflammation" that are presumed to represent proinflammatory responses are actually significantly increased in the presence of the M2a 
phenotype, which is often considered "antiinflammatory". This may suggest a re-consideration in the field as to what these immunohistochemical markers functionally represent.

Data on the effect of the M2a phenotype on amyloid deposition were inconclusive. We did find a decreasing trend in $A \beta$ levels in response to the AAV-IL-4 despite no marked statistical significance. This may have been due to the premature termination of the study. IL-4 has been studied in relation to AD pathology with mixed outcomes. Indeed, a study design very similar to the current study used AAV to overexpress IL-4 in the TgCRND8 mouse model. Here, increased amyloid deposition was observed 6 weeks post injection. Similar to the current study, enhanced GFAP and CD11b immunohistochemistry was also observed [13]. IL-4 has also been shown to attenuate $A \beta$-mediated neuroinflammation in a rat model [27] and promote the clearance of oligomeric $\mathrm{A} \beta$ by microglia in vitro [28]. The conflicting data highlight the complexities of the neuroinflammatory response and provide the rationale for further studies using better agents to chronically stimulate specific phenotypes.

Understanding the role of inflammation in Alzheimer's disease is accelerating within the field. Yet, the direction at which to target inflammation remains a hot topic of contention. Overall, the investigation accentuates that the microglial phenotypic continuum is influenced by multitude of dimensions. This study has emphasized that intricate cytokine networks function on a time- and concentration-dependent scale. Cytokine interplay needs to be further investigated in detail to maximize the therapeutic success of immune modulation. Further studies should address the heterogeneous inflammatory profile of the $\mathrm{AD}$ brain at different stages of progression across all etiologies and the physiological impacts of aging on microglial ability to retain their function. In addition, the complex communications between the differing glial cells of the CNS should be acknowledged when targeting the immune response. Taken together, research in this field should move toward elucidating a personalized therapeutic approach to treating individual $\mathrm{AD}$ patients.

\section{Conclusions}

Our data show that IL-4 induces a robust M2a phenotype in microglial cells in both in vitro and in vivo models with implicating elevations of M1 phenotypic markers. The M2a inflammatory state is associated with increased microgliosis and astrogliosis as evidenced by increased CD11b and GFAP-positive immunostaining. Additionally, biochemical analysis shows modest reductions in soluble and insoluble A $\beta$ lengths in APP/PS1sw transgenic mice at 3 months of age.

\section{Abbreviations}

AD: Alzheimer's disease; A $\beta$ : Beta-amyloid; IFNY: Interferon gamma; IL-1 $\beta$ : Interleukin 1 beta; TNFa: Tumor necrosis factor alpha; IL-12: Interleukin 12; IL-6: Interleukin 6; ARG1: Arginase 1; FcyR 1 and 3: Fc gamma receptor 1 and 3; TGF $\beta$ : Transforming growth factor beta; SPHK1: Sphingosine kinase 1; LPS: Lipopolysaccharide; AAV: Adeno-associated virus; SEM: Standard error of the mean; MRC1: Mannose receptor 1; YM1: CHL3, Chitin-like protein; CD86: Cluster of differentiation 86; $1 \times$ DPBS: Dulbecco's phosphate-buffered saline; ABC: Advidin-biotin complex; DAB: Diaminobenzidine; CA: Cornu ammonis; DG: Dentate gyrus; FCX: Frontal cortex; MPER: Mammalian protein extraction reagent; BCA: Bicinchonic acid; MSD: Meso-scale discovery; ELISA: Enzyme-linked immunosorbent assay; RT-PCR: Real-time polymerase chain reaction; ANOVA: Analysis of variance; GFAP: Glial fibrillary acidic protein.

\section{Competing interests}

The authors declare they have no competing interests.

\section{Authors' contributions}

$\mathrm{CHL}$ performed the data collection and analysis, interpreted the data and prepared the manuscript. TLS managed the animals in the study and assisted in data collection. ELA analyzed the data. GJP and MDM developed the $A A V$ vectors. $H M B$ assisted in preparation and revision of the manuscript. EMW, KB and FGO assisted in tissue processing and data collection. DMW conceived of the studies, analyzed and interpreted the data, and edited the final manuscript. All authors have approved the final version of this manuscript.

\section{Acknowledgements}

These studies were funded by NIH grant NS079637 (DMW) and NIH grant P20GM103486 (PI: L.B. Hersh, pilot grant awarded to DMW), assisted by the Viral Core of the COBRE in the Molecular Basis of Human Disease (NCRR 5P20RR020171, MDM and L.B. Hersh, co-PIs) and by the ADC Biostatistics Core (P3OAG028383). The contents of this manuscript are solely the responsibility of the authors and do not necessarily represent the official views of the $\mathrm{NIH}$, the NINDS or the NIGMS.

\section{Author details}

'Department of Physiology, University of Kentucky, Sanders-Brown Center on Aging, Lexington, KY 40536, USA. ${ }^{2}$ Department of Physiology, University of Kentucky, Lexington, KY 40536, USA. ${ }^{3}$ Department of Epidemiology, University of Kentucky, Lexington, KY 40536, USA. ${ }^{4}$ Department of Molecular and Cellular Biochemistry, University of Kentucky, Lexington, KY 40536, USA. ${ }^{5}$ Department of Biology, The University of Manchester, Manchester M13 9PL, UK. ${ }^{6}$ University of Kentucky, Room 424 Sanders-Brown Center on Aging, 800 S Limestone Street, Lexington, KY 40536, USA.

Received: 3 October 2014 Accepted: 10 January 2015

Published online: 04 March 2015

\section{References}

1. Alzheimer A, Stelzmann RA, Schnitzlein HN, Murtagh FR. An English translation of Alzheimer's 1907 paper, "Uber eine eigenartige Erkankung der Hirnrinde". Clin Anat. 1995;8:429-31.

2. Akiyama H, Barger S, Barnum S, Bradt B, Bauer J, Cole GM, et al. Inflammation and Alzheimer's disease. Neurobiol Aging. 2000;21:383-421.

3. Wilcock DM. A changing perspective on the role of neuroinflammation in Alzheimer's disease. Int J Alzheimers Dis. 2012;2012:495243.

4. Chan WY, Kohsaka S, Rezaie P. The origin and cell lineage of microglia: new concepts. Brain Res Rev. 2007;53:344-54.

5. Colton CA, Mott RT, Sharpe H, Xu Q, Van Nostrand WE, Vitek MP. Expression profiles for macrophage alternative activation genes in $A D$ and in mouse models of AD. J Neuroinflammation. 2006;3:27.

6. Colton CA, Wilcock DM. Assessing activation states in microglia. Neurol Disord Drug Targets. 2010;9:174-91.

7. Mosser DM. The many faces of macrophage activation. J Leukoc Biol. 2003;73:209-12.

8. Mantovani A, Sica A, Sozzani S, Allavena P, Vecchi A, Locati M. The chemokine system in diverse forms of macrophage activation and polarization. Trends Immunol. 2004;25:677-86. 
9. Edwards JP, Zhang X, Frauwirth KA, Mosser DM. Biochemical and functional characterization of three activated macrophage populations. J Leukoc Biol. 2006;80:1298-307

10. Mosser DM, Edwards JP. Exploring the full spectrum of macrophage activation. Nat Rev Immunol. 2008;8:958-69.

11. Gordon S, Martinez FO. Alternative activation of macrophages: mechanism and functions. Immunity. 2010;32:593-604.

12. Kiyota T, Okuyama S, Swan RJ, Jacobsen MT, Gendelman HE, Ikezu T. CNS expression of anti-inflammatory cytokine interleukin-4 attenuates Alzheimer's disease-like pathogenesis in APP + PS1 bigenic mice. FASEB J. 2010;24:3093-102

13. Chakrabarty P, Tianbai L, Herring A, Ceballos-Diaz C, Das P, Golde TE Hippocampal expression of murine $\mathrm{IL}-4$ results in exacerbation of amyloid deposition. Mol Neurodegener. 2012;7:36.

14. Jankowsky JL, Fadale DJ, Anderson J, Xu GM, Gonzales V, Jenkins NA, et al. Mutant presenilins specifically elevate the levels of the 42 residue beta-amyloid peptide in vivo: evidence for augmentation of a 42-specific gamma secretase. Hum Mol Genet. 2004;13:159-70.

15. Sudduth TL, Wilson JG, Everhart A, Colton CA, Wilcock DM. Lithium treatment of APPSWDI/NOS2-/- mice leads to reduced hyperphosphorylated tau, increased amyloid deposition and altered inflammatory phenotype. PLoS One. 2012;7:e31993.

16. Livak KJ, Schmittgen TD. Analysis of relative gene expression data using real-time quantitative PCR and the 2(-Delta Delta C(T)) Method. Methods. 2001;25:402-8.

17. Dik MG, Jonker C, Hack CE, Smit JH, Comijs HC, Eikelenboom P. Serum inflammatory proteins and cognitive decline in older persons. Neurology. 2005:64:1371-7.

18. Guerreiro RJ, Santana I, Bras JM, Santiago B, Paiva A, Oliveira C. Peripheral inflammatory cytokines as biomarkers in Alzheimer's disease and mild cognitive impairment. Neurodegener Dis. 2007:4:406-12.

19. Mandrekar-Colucci S, Landreth GE. Microglia and inflammation in Alzheimer's disease. CNS Neurol Disord Drug Targets. 2010;9:156-67.

20. Herber DL, Roth LM, Wilson D, Wilson N, Mason JE, Morgan D, et al. Time-dependent reduction in Abeta levels after intracranial LPS administration in APP transgenic mice. Exp Neurol. 2004;190:245-53.

21. Lee JW, Lee YK, Yuk DY, Choi DY, Ban SB, Oh KW, et al. Neuro-inflammation induced by lipopolysaccharide causes cognitive impairment through enhancement of beta-amyloid generation. J Neuroinflammation. 2008:5:37.

22. Shaftel SS, Griffin WS, O'Banion MK. The role of interleukin-1 in neuroinflammation and Alzheimer disease: an evolving perspective. J Neuroinflammation. 2008:5:7.

23. Gadani SP, Cronk JC, Norris GT, Kipnis J. IL-4 in the brain: a cytokine to remember. J Immunol. 2012;189:4213-9.

24. Block ML, Zecca L, Hong JS. Microglia-mediated neurotoxicity: uncovering the molecular mechanisms. Nat Rev Neurosci. 2007:8:57-69.

25. Kettenmann H, Hanisch UK, Noda M, Verkhratsky A. Physiology of microglia. Physiol Rev. 2011:91:461-553.

26. Weekman EM, Sudduth TL, Abner EL, Popa GJ, Mendenhall MD, Brothers HM, et al. Transition from an M1 to a mixed neuroinflammatory phenotype increases amyloid deposition in APP/PS1 transgenic mice. J Neuroinflammation. 2014;11:127.

27. Lyons A, Griffin RJ, Costelloe CE, Clarke RM, Lynch MA. IL-4 attenuates the neuroinflammation induced by amyloid-beta in vivo and in vitro. J Neurochem. 2007:101:771-81.

28. Shimizu E, Kawahara K, Kajizono M, Sawada M, Nakayama H. IL-4-induced selective clearance of oligomeric beta-amyloid peptide(1-42) by rat primary type 2 microglia. J Immunol. 2008;181:6503-13

\section{Submit your next manuscript to BioMed Central and take full advantage of:}

- Convenient online submission

- Thorough peer review

- No space constraints or color figure charges

- Immediate publication on acceptance

- Inclusion in PubMed, CAS, Scopus and Google Scholar

- Research which is freely available for redistribution

Submit your manuscript at www.biomedcentral.com/submit 